\title{
EDITORIAL
}

\section{AN AFFIRMATION OF OUR COMMITMENT TO STUDENT MEDICAL RESEARCH}

We are proud to present this issue of the $M J M$, which continues to generate the enthusiasm that inspired the journal's establishment. The publication of this issue affirms our original goal of producing a quality forum for disseminating students' contributions to medicine.

Since producing and distributing the premiere issue to readers throughout the world, we have created some exciting new developments in pursuit of this objective. Our authorship base has expanded to an international level to include students from the following institutions:

\author{
Dartmouth Medical School \\ Harvard Medical School \\ Jimma Institute of Health Sciences School of Medicine \\ Institut Curie \\ Laval University Medical Center \\ McGill University Faculty of Medicine \\ Queen's University Faculty of Medicine \\ School for International Training \\ University of Cambridge \\ University of Montreal Faculty of Medicine \\ University of Pennsylvania School of Medicine \\ University of Toronto Faculty of Medicine \\ University of Western Ontario Faculty of Medicine
}

We are looking forward to seeing this list grow with the publication of each new issue. Another accomplishment has been the creation of the electronic version of the $M J M$, which is currently available online. We encourage you to visit our site in the World Wide Web:

http://www.medcor.mcgill.ca/MJM/home.html. Finally, we have published a new "Authors' Section" in this issue containing information which explains the $M J M$ editorial review process for authors' manuscript submissions.

The articles contained in this issue reflect the broad scope of our publication, and validate our conviction that students can make important contributions to medical science. For example, the first two articles, epidemiological studies undertaken in Southwestern Ethiopia and Northeastern Brazil, illustrate the types of projects that students may pursue in community health. They remind us that the health of populations in developing countries would benefit greatly from the relatively straightforward implementation of inexpensive 
public health measures such as health education, sanitation, and immunization.

This issue's "MJM Focus," a section containing contributions from McGill faculty members and residents on a medical subspecialty or topic of interest, is on surgical approaches to the management of temporal lobe epilepsy (TLE). The contributions from Dr. William Feindel and Dr. Fernando Cendes illustrate how the persistent application of basic and clinical scientific knowledge toward solving recalcitrant clinical problems combined with the fertile exchange of ideas between specialists from diverse disciplines has been crucial for achieving success in treating TLE, a debilitating neurological condition; may this serve as a rousing paradigm for ongoing medical research efforts.

The quality and content of the $M J M$ depend on your continuing input. We encourage you to submit your comments and suggestions for ways we can improve. Our future efforts will be to expand the readership of both paper and electronic formats of the $M J M$, ensure the journal is placed in an appropriate reference index, and continue to develop the $M J M$ in response to our readers' needs.

At this formative stage of the $M J M$ 's development, we have already made much progress toward achieving our objectives. As the annual turnover of editors in leadership positions occurs to enable senior students to assume their clinical responsibilities, my role changes from editor-in-chief to editorial consultant following this issue. I and other members of the current editorial board have found producing the MJM to be an invaluable educational experience, and have greatly enjoyed our tenure. We look forward to working with the new editors whom we have trained to assume the leadership responsibilities that will ensure the continuity of the $M J M$. Under the capable stewardship of these individuals and those who will follow in their footsteps, the future of the McGill Journal of Medicine is bright, indeed.

I would like to thank all of the people who believed in the $M J M$ and made its publication possible. I would especially like to thank Allain, Laura, Joel, Andy, Jian-Zhuang, and Jong who are the founding senior staff members and have been dear friends and tireless coworkers throughout the realization of this project.

Jonathan E. Lim, M.S.

Editor-in-Chief

\section{BIOGRAPHY}

Jonathan E. Lim was the founding editor of the McGill Journal of Medicine. He received his B.S. and M.S. degrees in Biology from Stanford University (Stanford, California) in 1993 and is presently a third-year medical student at McGill University (Montreal, Quebec). His previous research interests have included Hox-B1 gene regulation at the Salk Institute for Biological Studies (La Jolla, California), cytokine activity in ocular tissues at McGill, and modulation of the corneal wound healing response after excimer laser photorefractive keratectomy at the Massachusetts Eye and Ear Infirmary, Harvard Medical School (Boston, Massachusetts).

Copyright (C) 1995 by MJM 